\title{
Effect of interlayer thickness on fatigue behavior in A5052 aluminum alloy with DLC/thermally sprayed WC-12Co hybrid coatings
}

\author{
Y. Uematsu ${ }^{1}$, T. Kakiuchi ${ }^{1}$, T. Teratani ${ }^{2}$, Y. Kobayashi ${ }^{2}$, \\ Y. Harada $^{2}$ \& M. Takekawa ${ }^{3}$ \\ ${ }^{1}$ Department of Mechanical Engineering, Gifu University, Japan \\ ${ }^{2}$ TOCALO Co., Ltd., Japan \\ ${ }^{3} Y K K$ Co., Ltd., Japan
}

\begin{abstract}
Tungsten carbide with $12 \%$ cobalt (WC-12Co) was thermally sprayed by a high velocity oxygen fuel (HVOF) method on A5052 aluminum alloy as an interlayer with the thicknesses of 25, 70 and $120 \mu \mathrm{m}$ and subsequently diamond-like carbon (DLC) was deposited with the thickness of $15 \mu \mathrm{m}$ to fabricate DLC/WC12Co hybrid coating. Rotary bending fatigue tests were conducted using the specimens with DLC/WC-12Co hybrid coating and the thickness effect of interlayer on fatigue behavior was investigated. The fatigue strengths of the specimens with WC-12Co single layer were higher than those of the substrate. The fatigue strengths increased with increasing the thickness of WC-12Co layer. The specimens with hybrid coating exhibited higher fatigue strengths than the specimens with WC-12Co single layer when the thickness of WC-12Co layer was the same. The thinnest WC-12Co interlayer led to the mostly improved fatigue strengths by hybrid coating system. In the specimens with hybrid coating, DLC film could suppress the micro-cracking of WC-12Co interlayer, and thus the fatigue strengths were improved. When the interlayer became thicker, the effect of thin DLC film became weaker and resulted in the lower improvement of fatigue strengths.
\end{abstract}

Keywords: fatigue, DLC, thermal spray, hybrid coating, crack initiation. 


\section{Introduction}

Aluminum (Al) alloys are widely used as light weight structural materials, while their wear resistance is lower than conventional steels. Diamond-like carbon (DLC) film is very hard and could be protective film against wear. In addition, it is known that hard coatings on the material could improve fatigue strengths and DLC films are effective for the enhancement of fatigue strengths in light weight alloys [1-3]. When DLC is directly deposited on the Al substrate, the delamination of DLC film easily occurs under high contact pressure because the elastic modulus of $\mathrm{Al}$ substrate is much lower than that of DLC film so that hard DLC film could not follow larger deformation of Al substrate. Therefore WC12Co was thermally sprayed as an interlayer between substrate and DLC film in order to improve the resistance against delamination under the contact pressure [4]. In the present study, the thickness of WC-12Co interlayer was changed as 25, 70 and $120 \mu \mathrm{m}$, keeping the thickness of DLC film constant as $15 \mu \mathrm{m}$. Using the specimens with hybrid coatings, rotating bending tests were conducted and the thickness effect of interlayer on the fatigue behavior was investigated.

\section{Experimental procedure}

\subsection{Materials and specimens}

The material was extruded round bar A5052-H14 aluminum alloy, and the hourglass-shaped specimens with a reduced section of $6 \mathrm{~mm}$ were machined from the bar. The mechanical properties of the material are $0.2 \%$ poof stress $\sigma_{0.2}$ : 213MPa, tensile strength $\sigma_{\mathrm{B}}$ : 252MPa, elastic modulus E: 86GPa, Vickers hardness: $81 \mathrm{HV}$. WC-12Co was thermally sprayed on the gauge section of the specimen by an HVOF method as an interlayer. The thickness of WC-12Co was 25, 70 or $120 \mu \mathrm{m}$. Subsequently DLC film, whose thickness was $15 \mu \mathrm{m}$, was deposited on the interlayer by a low-temperature plasma-enhanced chemical vapor deposition (PECVD) technique. The temperature during deposition was

Table 1: $\quad$ Fatigue specimen notation.

\begin{tabular}{c|c|c|c}
\hline \multicolumn{2}{c|}{ Specimen } & \multicolumn{2}{c}{ Coating } \\
\hline Category & Notation & WC-12Co & DLC \\
\hline \hline Substrate & Substrate & & \\
\hline DLC single & D15 & & $15 \mu \mathrm{m}$ \\
\hline \multirow{4}{*}{ WC-12Co single } & WC25 & $25 \pm 5 \mu \mathrm{m}$ & \\
\cline { 2 - 4 } & WC70 & $70 \pm 10 \mu \mathrm{m}$ & \\
\cline { 2 - 4 } & WC120 & $120 \pm 10 \mu \mathrm{m}$ & \\
\hline \multirow{2}{*}{$\begin{array}{c}\text { DLC/WC-12Co } \\
\text { hybrid }\end{array}$} & D15WC25 & $25 \pm 10 \mu \mathrm{m}$ & $15 \mu \mathrm{m}$ \\
\cline { 2 - 4 } & D15WC70 & $70 \pm 10 \mu \mathrm{m}$ & $15 \mu \mathrm{m}$ \\
\cline { 2 - 4 } & D15WC120 & $120 \pm 10 \mu \mathrm{m}$ & $15 \mu \mathrm{m}$ \\
\hline
\end{tabular}


$80^{\circ} \mathrm{C}$. Before DLC deposition, WC-12Co film surface was polished by emery paper with the grade of \#2000 for deflashing. The substrate specimens without coating and the specimens with WC-12Co or DLC single layer were also used in the fatigue tests for comparison. The thickness of WC12-Co single layer was 25, 70 or $120 \mu \mathrm{m}$, and that of DLC film was $15 \mu \mathrm{m}$. The specimen types and notations were summarized in Table 1.

\subsection{Test methods}

Elastic modulus and hardness of films were measured by a nano-indenter with Berkovich indenter whose radius of the tip was $20 \mathrm{~nm}$. The film properties were measured from surface to the depth of 500nm in DLC and to $1000 \mathrm{~nm}$ in WC12Co films. The fatigue tests were conducted by a cantilever-type rotary bending fatigue testing machine operating at a frequency of $19 \mathrm{~Hz}$.

\section{Experimental results}

\subsection{Appearance of films and mechanical properties}

The SEM micrographs showing the surfaces of D15 (DLC single layer), WC70 (WC-12Co single layer) and D15WC70 (hybrid coating) specimens are shown in Fig. 1. The surface of DLC film was flat and smooth, whilst WC-12Co single layer and hybrid coatings revealed rough surfaces. Figure 2 shows the cross sections of D15, WC70 and D15WC70 specimens. The interface between coating and film was very rough in WC70 because hard WC-12Co particles were

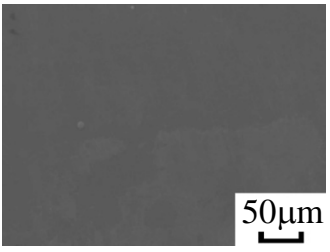

(a) D15

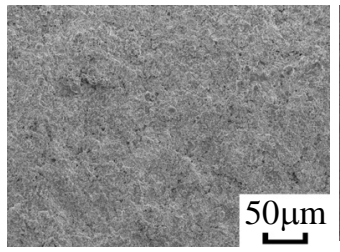

(b) WC70

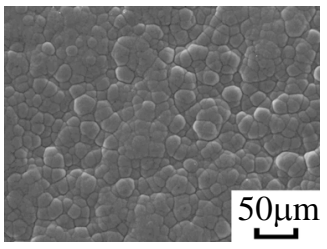

(c) D15WC70

Figure 1: $\quad$ SEM micrographs showing specimen surface.

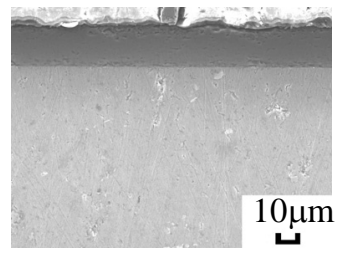

(a) D15.

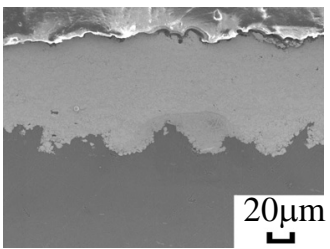

(b) WC70.

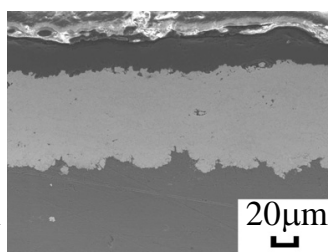

(c) D15WC70.

Figure 2: $\quad$ SEM micrographs showing cross section of coating. 
sprayed at high speed on the soft aluminum substrate (Fig. 2(b)). In the hybrid coating, the WC-12Co interlayer was completely covered by the DLC outerlayer as shown in Fig. 2(c), and rough interface between WC-12Co and substrate could be traced on the surface of DLC film. The elastic modulus and hardness of films measured by nano-indenter are revealed in Table 2. DLC was harder than WC-12Co, whilst the elastic modulus was lower. It should be noted that the properties of both films are much larger than those of the substrate.

Table 2: $\quad$ Mechanical properties of coating tested by nano-indenter.

\begin{tabular}{c|cc}
\hline Coating & $\begin{array}{c}\text { Elastic } \\
\text { modulus } \\
E(\mathrm{GPa})\end{array}$ & $\begin{array}{c}\text { Hardness } \\
(\mathrm{HV})\end{array}$ \\
\hline WC-12Co & 319 & 1368 \\
\hline DLC & 145 & 1630 \\
\hline
\end{tabular}

\subsection{Fatigue strength}

The $S$ - $N$ diagram is shown in Fig. 3, where the stress amplitude was calculated using the diameter including the thickness of coatings. The fatigue limit of the substrate was $130 \mathrm{MPa}$. As shown by the solid symbols, the fatigue strengths were improved by WC-12Co single layer and increased with increasing coating thickness. The $S-N$ diagram of the specimens with hybrid coatings is indicated by hollow symbols. The fatigue strengths were also improved with increasing the thickness of interlayer. When the thickness of the interlayer was $25 \mu \mathrm{m}$, the fatigue strengths were well improved by the DLC film on WC-12Co. However,

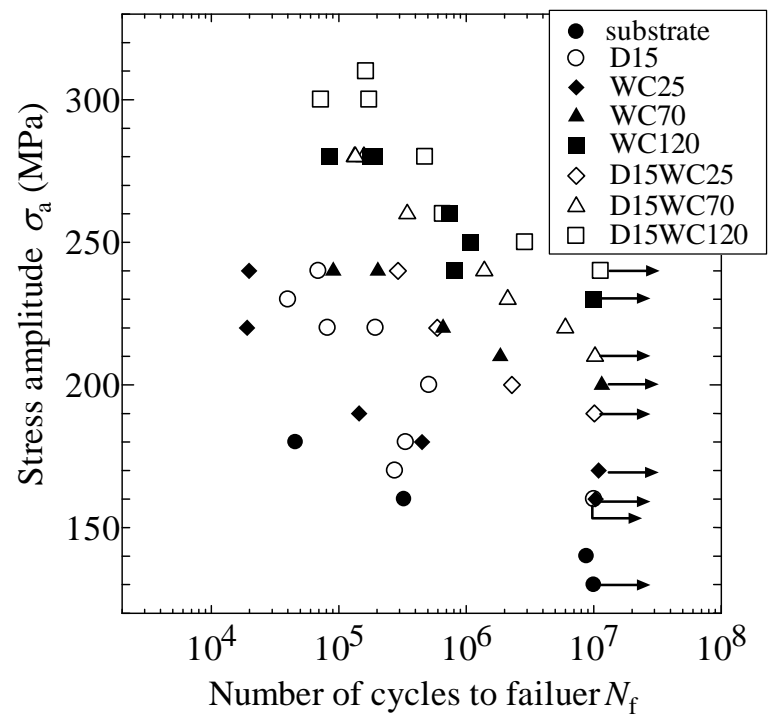

Figure 3: $\quad S-N$ diagram. 
the beneficial effect of DLC outerlayer was decreased with increasing the thickness of interlayer. The specimens with DLC single layer exhibited similar fatigue strengths with WC25.

\subsection{Fatigue crack initiation}

The fracture surfaces near the crack initiation sites of D15, WC70 and D15WC70 specimens are revealed in Fig. 4. In D15 specimen (Fig. 4(a)), the crack initiation site was flat and smooth, indicating that cyclic slip deformation in the substrate led to the fatigue crack initiation [2, 3]. As shown in Fig. 4(b) and (c), in WC70 and D15WC70 specimens, fatigue crack initiated at the small notch introduced during HVOF process as shown by the arrows in the figures. In the other specimens with WC-12Co single layer or hybrid coatings, fatigue cracks invariably initiated at the small notches. It indicates that the cracks were generated due to the stress concentration at the notches regardless of film thickness. It should be noted that DLC film does not peel off from the interlayer in Fig. 4(c), suggesting that the good adhesion of the film was achieved not only to the substrate but also to the interlayer.

In order to figure out fatigue crack initiation mechanism, WC70 specimen was fatigued at the stress amplitude of $240 \mathrm{MPa}$, until a fatigue crack was found on the specimen surface. In this procedure, crack initiation was detected using a digital microscope with the magnification of $x 1000$. As a result, at the very late stage of total fatigue life $\left(N / N_{\mathrm{f}} \approx 0.9\right.$ ), relatively long crack with the length of about $4 \mathrm{~mm}$ suddenly appeared on the surface. It implies that the fatigue crack initiated at the notch of substrate and grew to some extent, and subsequently brought about the sudden and unstable cracking of the coating.

The crack initiation behavior of the specimen with the hybrid coating, D15WC120, during the fatigue test was investigated by the acoustic emission (AE) method. In the AE method, HPS and LPS was set to be $100 \mathrm{kHz}$ and $2 \mathrm{MHz}$, respectively for the filtering. When the amplitude of $\mathrm{AE}$ was larger than $30 \mathrm{~dB}$, $\mathrm{AE}$ was counted as $1 \mathrm{Hit}$. AE counts during the fatigue test are shown in Fig. 5(a). The background noises with the amplitude of 30 40dB coming from mechanical contact were recognized during whole period of fatigue life, but AE

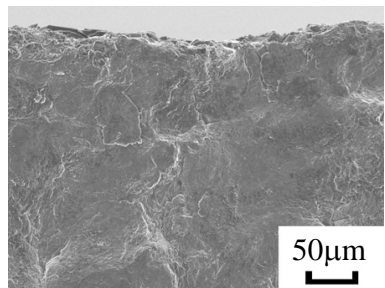

(a) D15.

$\left(\sigma_{\mathrm{a}}=180 \mathrm{MPa}, N_{\mathrm{f}}=3.3 \times 10^{5}\right)$

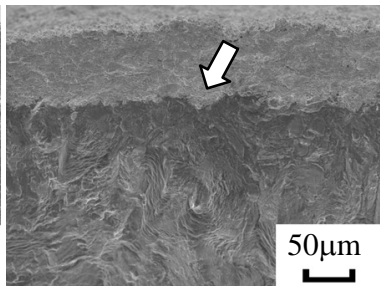

(b) WC70.

$\left(\sigma_{\mathrm{a}}=240 \mathrm{MPa}, N_{\mathrm{f}}=2.0 \times 10^{5}\right)$

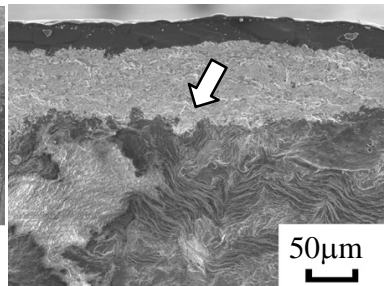

(c) D15WC70.

$\left(\sigma_{\mathrm{a}}=220 \mathrm{MPa}, N_{\mathrm{f}}=5.9 \times 10^{6}\right)$

Figure 4: $\quad$ SEM micrographs showing crack initiation site. 


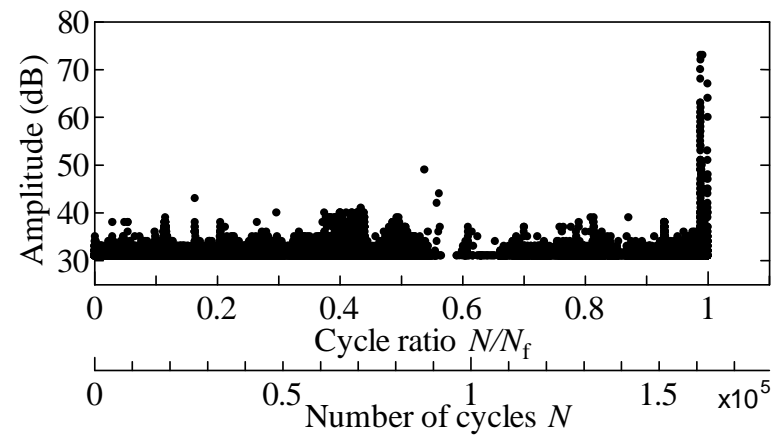

(a) $N / N_{\mathrm{f}}=0 \sim 1$.

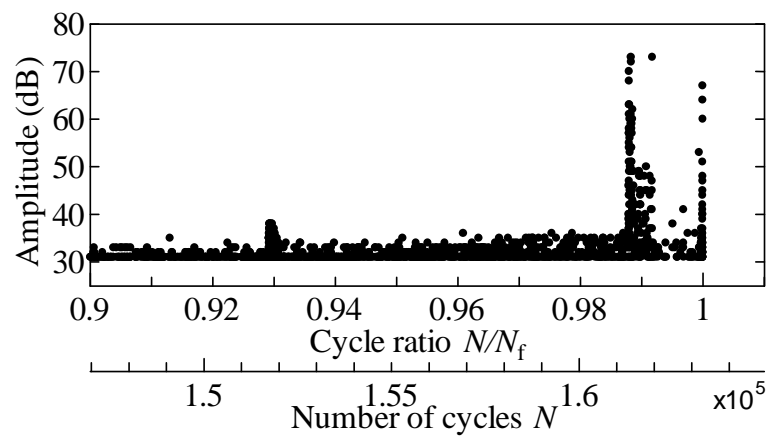

(b) $N / N_{\mathrm{f}}=0.9 \sim 1$.

Figure 5: AE amplitude vs. fatigue cycle ratio and number of cycles: (D15WC120, $\sigma_{\mathrm{a}}=310 \mathrm{MPa}, N_{\mathrm{f}}=1.6 \times 10^{5}$ ).

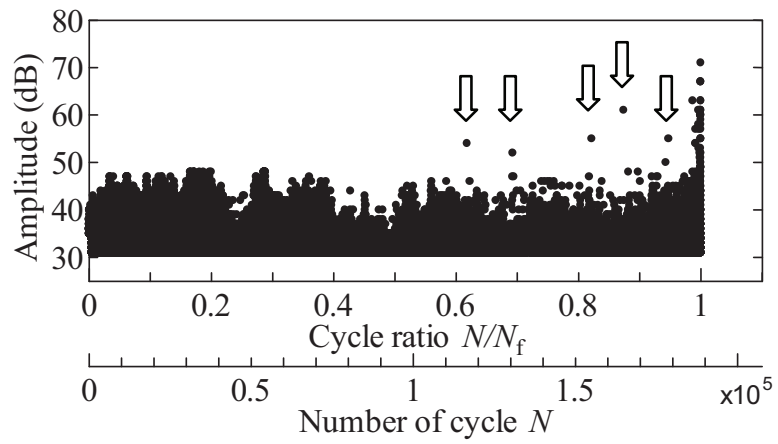

Figure 6: AE amplitude vs. fatigue cycle ratio and number of cycles: (WC120, $\sigma_{\mathrm{a}}=280 \mathrm{MPa}, N_{\mathrm{f}}=1.8 \times 10^{5}, N / N_{\mathrm{f}}=0 \sim 1$ ). 
counts with large amplitude was found near the end of the fatigue life where $N / N_{\mathrm{f}}$ $\approx 0.99$. AE hits counted in the period between $N / N_{\mathrm{f}} \approx 0.9$ and final fracture $\left(N / N_{\mathrm{f}}=1\right)$ are enlarged and shown in Fig. 5(b). AE hits with large amplitude were recognized just before the final fracture. These are typical AE hits for the specimens with hybrid coatings, where large AE hits occur only just before the final fracture, whilst AE hits were stable nearly whole period of fatigue test. Before the large AE hit, fatigue crack was not recognized, but the fatigue crack with the length of a few millimeter was found after the large AE hit by a digital microscope.

Figure 6 indicates AE hits of WC-12Co single layered specimen (WC120) during fatigue test. The mechanical noise was slightly larger than Fig. 5, but some AE hits with large amplitudes were found when $N / N_{\mathrm{f}}$ was larger than 0.6 as shown by the arrows.

\subsection{Subsurface fatigue crack front morphology}

The fatigue test was conducted at the stress amplitude of 310MPa using the specimen with hybrid coating (D15WC120), and the specimen surface was observed by a digital microscope intermittently at the intervals of 3000 5000 cycles. Figure 7(a) reveals fatigue crack observed on the specimen surface, namely on the DLC film surface, at $N=9.2 \times 10^{4}$ cycles, where the crack length was $4.1 \mathrm{~mm}$. It is noteworthy that a fatigue crack was not recognized 3000 cycles before, where $N=8.9 \times 10^{4}$ cycles. Subsequently, the DLC film was removed by polishing using diamond emery paper, and the crack was observed on the WC12Co surface as shown in Fig. 7(b). It is clear that the fatigue crack lengths in DLC film and WC-12Co layer are identical. The specimen was broken by quasistatic bending load in order to observe fatigue fracture surface in the substrate. Figure 7(c) shows the fracture surface, where a white solid line represents fatigue crack front. The fatigue crack front was identified as the boundary between flat area and dimple-covered area as shown in Fig. 7(d). The crack length on the substrate surface was $1.6 \mathrm{~mm}$ and shorter than that of DLC film and WC-12Co layer.

\section{Discussion}

\subsection{Fatigue failure mechanism of specimens with hybrid coating}

As shown in the section 3.4, fatigue crack appeared on the surface at very late stage of fatigue life, and crack length in the substrate was shorter than those in DLC film and WC-12Co layer. It indicates that firstly fatigue crack initiated at the stress concentration site in the substrate (Fig. 4(c)), and grew without cracking of films. When crack grew to some extent, and stress intensity factor of subsurface crack exceeded the fracture toughness of WC-12Co layer, unstable fracture of hybrid coating occurred and fatigue crack suddenly appeared on the surface near the end of total fatigue life. The unstable fracture of coating led to the longer crack length in the coating than in the substrate. The large AE hits near the end of fatigue life (Fig. 5(b)) correspond to the sudden fracture of the coatings. 


\subsection{Improvement of fatigue strengths by coatings}

DLC single layer could improve fatigue strength of the substrate. It had been reported that DLC film could increase fatigue strength of light weight metals [1 3]. In this case, it is considered that hard DLC film could suppress the cyclic slip deformation of substrate, and lead to the improvement of fatigue strengths. In the specimens with WC-12Co single layers, fatigue strengths increased with increasing the thickness. Hard WC-12Co layer could suppress the slip deformation of substrate as well as DLC film, whilst WC-12Co was thicker than DLC film. As shown in Table 2, not only the hardness but also the elastic

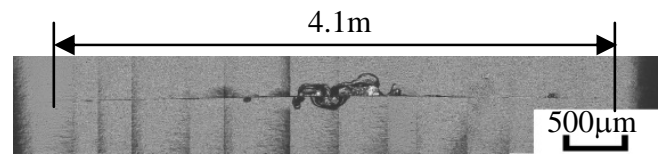

(a) Optical micrograph showing crack in DLC layer.

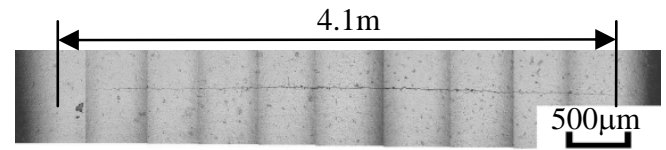

(b) Optical micrograph showing crack in WC-12Co interlayer.

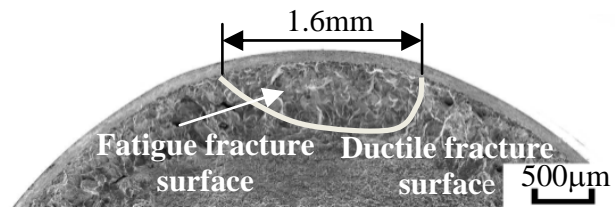

(c) SEM micrograph showing fracture surface in substrate with low magnification.

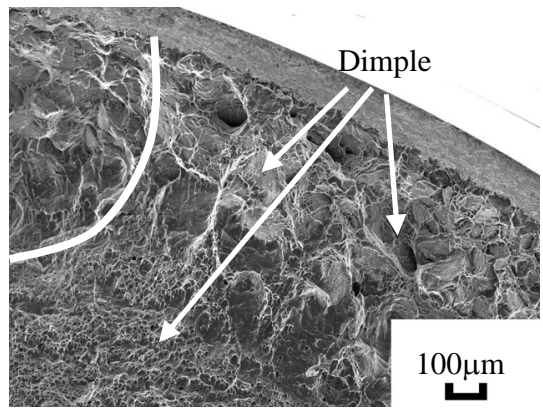

(d) SEM micrograph showing fracture surface in substrate with high magnification.

Figure 7: Crack feature at initial appearance on specimen's surface: (D15WC120, $\sigma_{\mathrm{a}}=310 \mathrm{MPa}, N=9.2 \times 10^{4}$ ). 
modulus were much higher than the substrate. Consequently, the fatigue load could be partly assigned to the thick WC-12Co layer with high elastic modulus, and relaxed the stress state in the substrate, resulting in the better fatigue strengths than the substrate. In the AE counts shown in Fig. 6, some AE hits with large amplitude were recognized prior to the final fracture, indicating that microcracking occurred in WC-12Co layer during fatigue loading.

In the specimens with hybrid coatings, fatigue strengths were further improved by the DLC outerlayer on the WC-12Co interlayer. Large AE hits were not recognized until $N / N_{\mathrm{f}} \approx 0.99$ as shown in Fig. 5 in contrast to the specimens with WC-12Co single layer (Fig. 6), indicating that the DLC film had suppressed the micro-cracking of WC-12Co layer. The fatigue damage in the interlayer such as micro-cracking could deteriorate the properties of WC-12Co layer. Consequently, the suppression of micro-cracking by DLC film is responsible for the better fatigue performances of the specimens with hybrid coatings than those with WC-12Co single layer. However, when the thickness of interlayer increases, namely, relative thickness of DLC film to the interlayer decreases, DLC film could not suppress the micro-cracking sufficiently because DLC film is getting thinner than the interlayer. Thus the beneficial effect by DLC film on the fatigue strengths decreased with increasing the thickness of the interlayer.

\section{Conclusions}

In this study, rotary bending fatigue tests had been conducted using specimens with DLC single layer, WC-12Co single layer or their hybrid coatings. The thickness of WC-12Co was changed and the thickness effects on the fatigue properties were discussed. The results obtained are as follows.

(1) Both DLC and WC-12Co single layers could improve fatigue strengths of the substrate. The fatigue strengths of the specimens with WC-12Co single layer increased with increasing the coating thickness.

(2) The fatigue strengths of the specimens with hybrid coatings were further improved comparing with the specimens with WC-12Co single layer. However, the beneficial effect of DLC film on the fatigue strengths decreased with increasing the thickness of the interlayer.

(3) The AE hits with large amplitude were recognized at the last stage of fatigue failure in the specimens with hybrid coatings, whilst some large $\mathrm{AE}$ hits were counted prior to the fatigue failure in the specimens with WC12Co single layer.

(4) DLC film on the WC-12Co interlayer could suppress the micro-cracking of interlayer, which led to the better fatigue strengths of the specimens with hybrid coatings. When the thickness of the interlayer increased, DLC film could not suppress the micro-cracking sufficiently because DLC film was getting thinner than WC-12Co interlayer. 


\section{References}

[1] Baragetti, S., Lusvarghi, L., Bolelli, G., Tordini, F., Surface and Coatings Technology, 203, pp. 3078-3087, 2009.

[2] Uematsu, Y., Tokaji, Takekawa, H., Fatigue and Fracture of Engineering Materials and Structures, 33, pp. 607-616, 2010.

[3] Uematsu, Y., Kakiuchi, T., Teratani, T., Harada, Y., Tokaji, K., Surface and Coatings Technology, 205, pp. 2778-2784, 2011.

[4] Okada, H., Uematsu, Y., Tokaji, K., Procedia Engineering, 2, pp. 283-290, 2010. 